\title{
Commentary on the "Co-Crystal of Tramadol-Celecoxib in Patients with Moderate to Severe Acute Post-Surgical Oral Pain: A Dose- Finding, Randomised, Double-Blind, Placebo- and Active-Controlled, Multicentre Phase II Trial". Implications for Cardiovascular Safety
}

\author{
Sebastián Videla ${ }^{1 *}$, Neus Gascón ${ }^{2}$, Carlos Plata-Salamán ${ }^{2}$
}

'Clinical Research Support Unit, Clinical Pharmacology Department, Bellvitge University Hospital/IDIBELL, Barcelona, Spain. ${ }^{2}$ Medical Sciences Area, ESTEVE Pharmaceuticals S.A., Barcelona, Spain.

\section{Article Info}

\section{Article Notes}

Received: December 4, 2018

Accepted: January 23, 2019

\section{${ }^{*}$ Correspondence:}

Dr. Sebastián Videla, Clinical Research Support Unit, Clinical Pharmacology Department, Bellvitge University Hospital/IDIBELL, Barcelona, Spain; Telephone No: +34 93446 6000; Email: svidelaces@gmail.com, svidela@ bellvitgehospital.cat.

C) 2019 Videla S. This article is distributed under the terms of the Creative Commons Attribution 4.0 International License.
Pain is the most common symptom for which patients seek medical attention and represents a major clinical, social, and economic problem across all ages, with estimates of the monthly prevalence ranging from $1.0 \%$ to over $60.0 \%{ }^{1}$. Acute and chronic pain management continues to be a challenge for physicians and presents a significant global unmet medical need. Often, pain relief is not adequately achieved ${ }^{2}$. In order to address this unmet medical need, the aim is to achieve rapid, effective and well-tolerated pain relief in the short term, and to maintain effective and well-tolerated pain control over the long term. Strategies of improving pain relief include multimodal analgesia, achieved via the use of various classes of pain-relieving drugs that have complementary mechanisms of action ${ }^{3}$.

Co-crystals are defined as "solids that are crystalline single phase materials composed of two or more different molecular and/or ionic compounds generally in a stoichiometric ratio which are neither solvates nor simple salts" ${ }^{\prime 4}$. Co-crystals represent a way to improve the properties of an active pharmaceutical ingredient (API) ${ }^{5}$. Cocrystals containing more than one API represent unique molecular structures that offer a novel approach to polypharmacology ${ }^{6}$. APIAPI co-crystals have the potential for improved physicochemical properties compared with their constituent drugs or their combinations, which may translate into clinical benefits. These may be apparent as enhanced solubility or dissolution characteristics, which in turn may improve the pharmacokinetic (PK) profile compared with open or traditional fixed-dose combinations ${ }^{6}$.

The co-crystal of tramadol-celecoxib (CTC) is a medical product based on a co-crystal molecule of two APIs with complementary mechanisms of action (tramadol and celecoxib) in a 1:1 molecular ratio (1:1.27 weight ratio), conferred by the intrinsic stoichiometry of the co-crystal structure ${ }^{7}$. A $100 \mathrm{mg}$ dose of this co-crystal contains $44 \mathrm{mg}$ of racemic tramadol hydrochloride (rac-tramadol. $\mathrm{HCl}$ ) and 56 mg of celecoxib. In the USA, tramadol is indicated for the management of pain in adults that is severe enough to require an opioid analgesic and for which alternative treatments are inadequate ${ }^{8}$. Tramadol is also marketed outside of the US ${ }^{9}$ and Europe where the indication varies between these two examples. 
In the USA, celecoxib in indicated for the management of signs and symptoms of osteoarthritis, rheumatoid arthritis, juvenile rheumatoid arthritis (in patients 2 years and older) and ankylosing spondylitis; celecoxib is also indicated for the management of acute pain and primary dysmenorrhea ${ }^{10}$. In Europe, celecoxib is indicated in adults for the symptomatic relief in the treatment of osteoarthritis, rheumatoid arthritis and ankylosing spondylitis.

Our group has recently published the phase II clinical trial results for $\mathrm{CTC}^{11}$. This was a dose-finding, single dose, double-blind, randomised, placebo- and active-controlled, parallel group study in patients with moderate-to-severe acute pain following oral surgery involving extraction of two or more impacted third molars, requiring bone removal. This was the first publication presenting efficacy and safety results to the scientific community on this new medical entity. Previously, phase I clinical trials: single dose $^{12}$, multiple doses ${ }^{13}$ and food interaction ${ }^{14}$, consistently showed a modified clinical PK profile of the CTC constituent moieties: slower absorption of tramadol with an important reduction of the Cmax (maximum serum concentration) without decreasing overall exposure, and faster absorption of celecoxib but without enhancing overall exposure.

This co-crystal effect on the CTC PK profile had a significant impact on efficacy. Findings from the phase II clinical trial support the concept that the unique molecular structure of the co-crystal form can confer advantageous physicochemical properties to the component APIs, which can ultimately translate into clinical benefits: a higher pain relief with lower doses. CTC 100, 150, and $200 \mathrm{mg}$ administration was associated with a significantly increased acute pain relief than tramadol $100 \mathrm{mg}$ and an improved (CTC 100 and $150 \mathrm{mg}$ ) or similar (CTC 200 $\mathrm{mg}$ ) safety profile. Gastrointestinal disorders were the most frequent treatment-related adverse events. Nausea, vomiting and dizziness were markedly less frequent with 100 and $150 \mathrm{mg}$ CTC than with tramadol $100 \mathrm{mg}$. Tramadol in CTC did not potentiate adverse events of celecoxib and vice versa. Thus, the improved efficacy with CTC occurred while maintaining a similar or improved safety profile. Hence, the co-crystal mechanism conferred by CTC appears to translate to improved efficacy achieved with lower amounts of each active moiety, a similar or improved safety profile and overall an enhanced benefit-to-risk ratio.

Regarding cardiovascular safety, a retrospective analysis of adverse drug reactions from the safety database Vigibase (the WHO global individual case safety report database system) did not identify an increased risk for any specific potential safety concern when both tramadol and celecoxib are administered concomitantly ${ }^{15}$, and to date, CTC clinical data including from the phase II clinical trial do not show any signal of concern. A risk management plan has been scheduled to provide evidence on the CTC safety profile and data are being collected in the phase III CTC clinical program.

In order to put CTC safety (including cardiovascular profile) in context, it is noteworthy to highlight that:

1) the therapeutic posology of CTC for moderate to severe pain is $100-200 \mathrm{mg}$ of CTC twice a day. These daily doses correspond to a maximum dose of 88-176 mg per day of tramadol and 112-224 mg per day of celecoxib. These are low doses in comparison to the recommended doses for acute pain when they are used as a single product,

2) new data on cardiovascular events from the PRECISION trialalso have to be taken into account. This was a randomized, multicenter, double-blind, non-inferiority trial involving patients who were at increased cardiovascular risk and had rheumatoid arthritis or osteoarthritis ${ }^{16}$. The goal of the trial was to assess the non-inferiority of celecoxib with regard to the primary composite outcome of cardiovascular death (including hemorrhagic death), nonfatal myocardial infarction, or non-fatal stroke. After a long period of treatment, celecoxib, ibuprofen, and naproxen had similar rates of cardiovascular events $(2.3 \%, 2.5 \%$, and $2.7 \%$, respectively). In contrast, the risk of gastrointestinal events was significantly lower with celecoxib than with naproxen $(\mathrm{P}=0.01)$ or ibuprofen $(\mathrm{P}=0.002)^{16}$.

In brief, the results of the phase II CTC clinical trial; showed a statistically significant and clinically meaningful acute pain relief associated with an improved safety profile at doses of CTC containing $100 \mathrm{mg}$ (44 mg tramadol plus $56 \mathrm{mg}$ celecoxib) and $150 \mathrm{mg}$ (66 mg tramadol plus 84 $\mathrm{mg}$ celecoxib) compared to tramadol $100 \mathrm{mg}$. CTC 200 $\mathrm{mg}$ produced the greatest pain relief with a similar safety profile, compared with tramadol $100 \mathrm{mg}$. The promising results of this phase II, randomized, single dose, doubleblind, regulatory clinical trial have to be validated in the planned phase III, randomized, multiple dose, doubleblind, active- and placebo-controlled, regulatory clinical trials.

The data suggest that the clinical benefits of CTC result from a dual mode of integrated action: 1) the optimized safety- and efficacy-enabling PK profile of each API conferred by the co-crystal mechanism, which works in tandem with

2) complementary molecular mechanisms for pain relief activated by each of the 3 active moieties in CTC acting in central and peripheral targets: tramadol (+)-enantiomer weak mu agonist and inhibitor of serotonin reuptake, tramadol (-)-enantiomer inhibitor of norepinephrine reuptake ${ }^{17}$, and celecoxib inhibitor of cyclo-oxygenase-2. All these molecular mechanisms, working together due to synchrony produced by the co-crystal mechanism, implies a pharmacodynamic efficacy synergism at lower doses. 


\section{Conflict of Interest}

SV was an employee of ESTEVE Pharmaceuticals S.A. during the clinical trial execution. $\mathrm{NG}$ and $\mathrm{CP}$ are employees of ESTEVE Pharmaceuticals S.A.

The study was funded by ESTEVE Pharmaceuticals, S.A.

\section{References}

1. Henschke N, Kamper SJ, Maher CG. The Epidemiology and Economic Consequences of Pain. Mayo Clinic Proceedings. 2015; 90(1): 139147.

2. WHO Guidelines for the pharmacological treatment of persisting pain in adults with medical illnesses. Available at: http://www. who.int/medicines/areas/quality_safety/Scoping_WHO_GLs PersistPainAdults_webversion.pdf?ua=1 [Last accessed October, 2018].

3. Chou R, Gordon DB, de Leon-Casasola OA, et al. Management of postoperative pain: a Clinical practice guideline from the American Pain Society, the American Society of Regional Anesthesia and Pain Medicine, and the American Society of Anesthesiologists' Committee on Regional Anesthesia, Executive Committee, and Administrative Council. J Pain. 2016; 17: 131-57.

4. Aitipamula S, Banerjee R, Bansal AK, et al. Polymorphs, salts and cocrystals: What's in a name? Cryst Growth Des. 2012; 12: 2147-52.

5. Duggirala NK, Perry, ML, Almarsson O, et al. Pharmaceutical cocrystals: along the path to improved medicines. Chem Comm. 2016; 52: 640-655.

6. Thipparaboina R, Kumar D, Chavan RB, et al. Multidrug co-crystals: towards the development of effective therapeutic hybrids. Drug Discov Today. 2016; 21: 481-90.

7. Almansa C, Mercè $\mathrm{R}$, Tesson $\mathrm{N}$, et al. Co-crystal of tramadol hydrochloride-celecoxib (ctc): A novel API-API co-crystal for the treatment of pain. Cryst Growth Des. 2017; 17: 1884-1892.

8. U.S FDA. Ultram ${ }^{\circledR}$ prescribing information. Available at: https://www. accessdata.fda.gov/drugsatfda_docs/label/2018/020281s042s043lbl.pdf [Last accessed October, 2018].

9. MHRA. Summary of product characteristics for Tramadol $50 \mathrm{mg}$ capsule 2015. Available at: http://www.mhra.gov.uk/home/groups/ spcpil/documents/spcpil/con1532664405478.pdf [Last accessed October, 2018].

10. U.S. FDA. Celebrex prescribing information 2018. Available at: www. accessdata.fda.gov/drugsatfda_docs/label/2018/020998s050lbl.pdf [Last accessed October, 2018].

11. López-Cedrún J, Videla S, Burgueño M, et al. Co-crystal of tramadolcelecoxib in patients with moderate to severe acute post-surgical oral pain: A dose-finding, randomised, double-blind, placebo- and activecontrolled, multicentre, phase II trial. Drugs R D. 2018; 18: 137-148.

12. Videla S, Lahjou M, Vaqué A, et al. Single-dose pharmacokinetics of cocrystal of tramadol-celecoxib: Results of a four-way randomized openlabel phase I clinical trial in healthy subjects. Br J Clin Pharmacol. 2017; 83: 2718-2728.

13. Videla S, Lahjou M, Vaqué A, et al. Pharmacokinetics of multiple doses of co-crystal of tramadol-celecoxib: findings from a four-way randomized open-label phase I clinical trial. Br J Clin Pharmacol. 2018; 84: 64-78.

14. Encina G, Encabo M, Escriche M, et al. The effect of food on tramadol and celecoxib bioavailability following oral administration of cocrystal of tramadol-celecoxib (CTC): A randomised, open-label, single-dose, crossover study in healthy volunteers. Clin Drug Investig. 2018. doi: 10.1007/s40261-018-0672-y.

15. Vaqué A, Sust M, Gascón N, et al. A review of safety data from spontaneous reports on marketed products containing tramadol and celecoxib: A Vigibase descriptive analysis. Adv Pharmacoepidemiol Drug Saf. 2014; 3: 164. doi:10.4172/2167-1052.1000164.

16. Nissen SE, Yeomans ND, Solomon DH, et al. Cardiovascular safety of celecoxib, naproxen, or ibuprofen for arthritis. N Engl J Med. 2016; 375: 2519-29.

17. Grond S, Sablotzki A. Clinical pharmacology of tramadol. Clin Pharmacokinet. 2004; 43(13): 879-923. 